\section{G263(P) THE TREATMENT OF ANOREXIA NERVOSA IN ADOLESCENTS: A METASYNTHESIS OF PARENTS' PERSPECTIVES}

1,20N Oketah, ${ }^{2} \mathrm{P}$ Frost. ${ }^{1}$ Department of Paediatrics and Adolescent Medicine, University College London, Hospital, UK; ${ }^{2}$ Postgraduate Medical School, University of Brighton and Sussex Medical School, Brighton, UK

\subsection{6/archdischild-2020-rcpch.227}

Background Anorexia nervosa (AN) is a severe eating disorder with onset typically in adolescence characterised by a distorted body image major and an intense fear of weight gain. It greatly impacts on adolescent growth, puberty and psychological development and is known to have the highest mortality of any psychiatric illness. Treatment is challenging and studies have established the central role of the family in recognition, treatment and recovery from anorexia.

Objectives The aim of this research was to perform a systematic review of all qualitative studies done on the views of parents on the treatment and recovery process of anorexia nervosa in their adolescent child.

Methods A systematic search of six databases: Medline, PsychINFO, CINHAL, EMBASE, Cochrane library and SSCI was conducted for qualitative studies regarding parents' views about the treatment of anorexia nervosa in adolescents. Quality of articles was assessed using the critical appraisal skills program (CASP). The chosen studies were analysed and key themes identified using thematic synthesis.

Results In total, the systematic search yielded 315 papers of which 14 studies from six countries met my inclusion criteria reporting the views of 202 parents. Four themes were developed from the analysis: Understanding the child and the disease, Experience of services and treatment modalities, the role of professionals and the concept of recovery.

Conclusion This is the first systematic review performed so far focussing solely on parents views on adolescent AN treatment and further highlights the importance of taking into account parents views in the treatment of adolescent anorexia. While they report relief in finding help at a very difficult time in their life, they continue to struggle with delays in finding help, judgmental attitudes of professionals and uncertainty about the future. Recognition by clinicians of the challenges faced by parents and families will help to provide better support, and a more holistic approach and hopefully reduce the burden of chronicity of adolescent anorexia into adulthood.

\section{G264(P) CHILDREN'S MENTAL HEALTH WEEK - PROMOTING CHILDREN'S MENTAL WELL-BEING IN HOSPITAL}

B Cuellar, E Morgan, S Reynolds. Women's and Children's, UH Bristol NHS Trust, Bristol, UK

10.1136/archdischild-2020-rcpch.228

Aims

- Raise awareness of ways you can look after your mental health and encourage people to think about practical ways to do so.

- Encourage people to talk about mental health and wellbeing

- Promote resources that can help with wellbeing and signpost to further support

Method Information boards and resource packs were developed that could be used on wards alongside the facilitated stand in the Children's Hospital reception. The resources included information about local city based mental health organisations.

The structure of the week included a different focus for each day; physical activity, diet, relationships and sleep. This was to ensure the team could bring in normal day to day activities and associate these with positive mental wellbeing.

Information boards included signposting, resources to take away and a question based activity.

In advance of the week information and plans were shared with ward sisters, matrons and ward mental health champions to ensure staff were aware of the plans.

The area in reception had the same resources as wards but was staffed by teams from throughout the hospital including physiotherapists, psychology and dieticians.

Results Feedback from the event was positive. One young governor who was involved shared how it was brilliantly received by staff and parents. There was lots of interest in how to keep yourself happy and healthy which was great to see!'

A comment received in feedback from a staff member who helped facilitate the stand shared 'It felt very much like a mental health drop in clinic that provided a lot of advice in a short space of time. It felt helpful and is a service that is currently not available to parents.

Conclusion The creation of opportunities to talk about mental health has been shown to be important both in engaging staff alongside reducing stigma and encouraging conversations with patients and families.

The use of information stands in reception and on wards was beneficial. It allowed engagement on the opportunities and provided informal support to families. The use of themes that linked practical and mental health was feedback as being particularly positive and gave a non-threatening way to engage in conversations for some people.

\section{G265(P) EVALUATION OF THE 'HEAR GLUE EAR' APP TO SUPPORT CHILDREN WITH CHRONIC OTITIS MEDIA WITH EFFUSION}

${ }^{1} S$ Fordington Fowkes, ${ }^{2} \mathrm{~T}$ Holland Brown. ${ }^{1}$ School of Clinical Medicine, University of Cambridge, Cambridge, UK; ${ }^{2}$ Cambridge Community Paediatrics, Cambridge NHS FT, Cambridge, UK

\subsection{6/archdischild-2020-rcpch.229}

Background Otitis Media with Effusion (OME), or Glue Ear, occurs in 1 in 5 pre-school children. This causes deafness at a critical developmental time, potentially affecting speech, learning and social development. Current practice involves a 'watchful waiting' period before grommets are considered. Studies show that parents feel unable to support their child during this period. 'Hear Glue Ear' is a novel app designed to provide reliable information on children's hearing progress as well as targeted activities to minimise developmental delay while they have OME.

Aims

- To evaluate acceptability and usability of the app for:

- Children with/without OME

- Parents/carers

- Clinicians in community paediatrics

- To assess the accuracy of the app's hearing screen to monitor hearing from home. 
Method We studied 61 2-8 year-old children (including 7 with Down's syndrome) attending audiology clinics with OME $(n=38)$ and normal hearing $(n=23)$. Children and parents were observed and questioned during their first use of the app to assess acceptability and usability. One week later, they were contacted about home use. Additionally, 18 clinicians completed questionnaires regarding use of the app in managing OME.

Results $80 \%$ of children liked the app and $90 \%$ found it easy to use. In children with Down's Syndrome, accessibility depended on developmental age and previous app experience rather than chronological age.

$89 \%$ of parents wanted their child to use the app regularly. Despite finding no significant correlation between the app's hearing screen and audiology clinic results $(p=0.381)$, $80 \%$ of parents felt more confident gauging and communicating their child's hearing levels. $89 \%$ of families used the app at home and $75 \%$ felt it helped their child's speech development.

$78 \%$ of clinicians would like patients to use the app regularly, and $89 \%$ believed it would help parents support their child's development.

Conclusions The 'Hear Glue Ear' app is acceptable to children, parents and clinicians. It offers an affordable and accessible means to monitor hearing at home, deliver speech and language therapy remotely, and support speech development during the 'watchful waiting' period of OME management.

\section{G266(P) A REGIONAL QUALITY IMPROVEMENT PROJECT FOR CONGENITAL CYTOMEGALOVIRUS (CCMV) ASSOCIATED SENSORINEURAL HEARING LOSS LEADING TO IMPROVED PRIORITISATION OF FINITE RESOURCES DURING THE COVID-19 PANDEMIC}

${ }^{1} \mathrm{Z}$ Khan, ${ }^{2} \mathrm{R}$ Brown, ${ }^{3} \mathrm{~V}$ Banks, ${ }^{4} \mathrm{~T}$ Holland Brown. ${ }^{1}$ University of Cambridge School of Medicine; ${ }^{2}$ Paediatrics, Addenbrooke's Hospital, Cambridge; ${ }^{3}$ Newborn Hearing Screening, Addenbrooke's Hospital, Cambridge; ${ }^{4}$ Community Paediatrics, Cambridge Community Services NHS Trust, Cambridge, UK

\subsection{6/archdischild-2020-rcpch.230}

Background Congenital CMV [cCMV] is a leading cause of hearing loss and childhood disability. Recent research demonstrated children's long-term outcomes could be improved with antiviral treatment offered within the first month of life. During the COVID-19 pandemic, national hearing screening guidance promoted prioritisation of face-to-face diagnostic auditory brainstem response $[A B R]$ in infants with cCMV.

Aim To identify babies with cCMV and hearing loss within 1 month of life to ensure:

- Greater certainty of the diagnosis of cCMV.

- Early paediatric referral for identification and management of cCMV

- Timely consideration of treatment

Method A truly joined-up, multidisciplinary approach was taken across the region (newborn hearing screeners, audiologists, ENT surgeons, virologists, neonatologist, paediatricians, ophthalmologists). Targeted cCMV testing was undertaken by newborn hearing screeners in all babies who 'failed' their otoacoustic emission and automated ABR. CMV positive babies were referred urgently to paediatricians to consider management and treatment within 1 month of life. We derived data on the timing of cCMV testing in the months before and then during the pandemic.

Results Regional hospitals worked together to write the East of England CMV guidelines which were then ratified. https:// www.networks.nhs.uk/nhs-networks/eoe-neonatal-odn/guidelines/ current-guidelines/congenital-cmv-guideline

Following implementation in early 2019, Addenbrookes Hospital data showed that babies undergoing hearing screening during the pandemic could be prioritised according to national guidance for definitive ABR testing

Conclusion CMV saliva swabs for babies born with hearing loss was implemented across the East of England region using a wide multidisciplinary approach. In the pandemic, this enabled prioritisation of diagnostic ABR testing in babies with $\mathrm{cCMV}$ and hearing impairment according to national guidance.

\section{Down syndrome medical interest group, british association of community child health and british paediatric respiratory society}

\section{G267 PREVENTING INFECTION AND MORTALITY IN DOWN SYNDROME - THE NOTTINGHAM EXPERIENCE}

${ }^{1,2}$ AP Prayle, ${ }^{1} P$ Shires, ${ }^{3} V$ Baicher, ${ }^{1} S$ Smith, ${ }^{3} L$ Walton, ${ }^{4} E$ McDermott, ${ }^{1} E$ Marder. ${ }^{1}$ Family Health, Nottingham University Hospitals NHS Trust, Nottingham, UK; ${ }^{2}$ Child Health, The University of Nottingham, Nottingham, UK; ${ }^{3}$ Emergency Department, Nottingham University Hospitals NHS Trust, Nottingham, UK; ${ }^{4}$ Immunology, Nottingham University Hospitals NHS Trust, Nottingham, UK

\subsection{6/archdischild-2020-rcpch.231}

Purpose Children with Trisomy 21 are at increased risk of death compared to age-matched controls. We recently undertook a retrospective review of mortality in Trisomy 21 in our tertiary non-cardiac centre, and found an infective cause of death in $9 / 16$ cases.

Methods In response to these data, we have undertaken a number of interventions at the Trust level with the goal of reducing the risk of future mortality and morbidity.

- New local guidance for preventing infection in Trisomy 21

- Aggressively treating comorbidities such as reflux and sleep related breathing disorders

- Protocol driven assessment of immune function including functional antibodies and meticulous immunisation

- We recommend influenza and pneumovax II for all

- An alert on the Trust wide computer system for patients when they present in the emergency department or on the Children's Assessment Unit to emphasise the risk of infection in Trisomy 21

- A review of how often and why children with Trisomy 21 present to ED

- Increasing awareness for parents, including an alert in the Down syndrome insert for the parent held child health record (the 'red book')

- A standardized alert sentence on all letters to primary care from the Trisomy 21 clinic emphasizing the risk of infection

- Increasing awareness amongst Clinicians, through programmed teaching, bulletins, and alerts 